\title{
Papers
}

\section{Effects of transfusion with red cells filtered to remove leucocytes: randomised controlled trial in patients undergoing major surgery}

Joost A van Hilten, Leo M G van de Watering, J Hajo van Bockel, Cornelis J H van de Velde, Job Kievit, Ronald Brand, Wilbert B van den Hout, Robert H Geelkerken, Rudi M H Roumen, Ronald M J Wesselink, Ankie W M M Koopman-van Gemert, Jan Koning, Anneke Brand for Tactics (transfusion associated complications study)

\begin{abstract}
Objective To compare postoperative complications in patients undergoing major surgery who received non-filtered or filtered red blood cell transfusions.

Design Prospective, randomised, double blinded trial.

Setting 19 hospitals throughout the Netherlands (three university; 10 clinical; six general).

Participants 1051 evaluable patients: 79 patients with ruptured aneurysm, 412 patients undergoing elective surgery for aneurysm, and 560 undergoing gastrointestinal surgery. Interventions The non-filtered products had the buffy coat removed and were plasma reduced. The filtered products had the buffy coat removed, were plasma reduced, and filtered before storage to remove leucocytes.

Main outcome measures Mortality and duration of stay in intensive care. Secondary end points were occurrence of multi-organ failure, infections, and length of hospital stay. Results No significant differences were found in mortality (odds ratio for filtered $v$ non-filtered $0.80,95 \%$ confidence interval 0.53 to 1.21$)$ and in mean stay in intensive care $(-0.4$ day, -1.6 to 0.6 day). In the filtered group the mean length of hospital stay was 2.4 days shorter $(-4.8$ to 0.0 day; $\mathrm{P}=0.050$ ) and the incidence of multi-organ failure was $30 \%$ lower (odds ratio $0.70,0.49$ to $1.00 ; \mathrm{P}=0.050$ ). There were no differences in rates of infection $(0.98,0.73$ to 1.32$)$.

Conclusion The use of filtered transfusions in some types of major surgery may reduce the length of hospital stay and the incidence of postoperative multi-organ failure.
\end{abstract}

\section{Introduction}

Red blood cell transfusions are indispensable in major surgery, but they still entail potential risks for morbidity and mortality. ${ }^{1-8}$ Although blood products have never been safer with respect to transmission of infectious diseases, patients who receive blood transfusions can still develop immunological reactions. Antibodies to leucocytes received during transfusions can cause febrile reactions which can be prevented by removal of leucocytes from the blood product. On the other hand, beneficial effects such as better survival of allografts in kidney transplantation ${ }^{9}$ and improved survival in patients with HIV who receive transfusions $^{10}$ are attributed to the allogeneic leucocytes.

Over the past decade, several studies have examined the possibility of an increased risk of postoperative complications associated with perioperatively transfused leucocytes. Specific issues addressed were the increased risks of postoperative infections and of recurrence of cancer after curative resection. The recurrence of cancer was explored in only one study in patients with colorectal cancer, in which there was no advantage of removing leucocytes from transfusions. ${ }^{11}$ Five prospective randomised studies investigated the incidence of postoperative infections after abdominal surgery by ${ }^{11-15}$ comparing outcome after transfusions of non-filtered red blood cells or red blood cells with leucocytes filtered out. Three of these trials ${ }^{13-15}$ observed a reduction and two ${ }^{11}{ }^{12}$ found a similar incidence of postoperative infections after filtered transfusions. Heterogeneity prohibited calculation of a common odds ratio for these trials. ${ }^{16}$ In the only study performed in cardiac surgery, filtered products were associated with a reduction in postoperative infections and mortality due to multi-organ failure, but these effects were present only in patients who had received 4 or more units. ${ }^{17}$ Multi-organ failure is a common postoperative complication after major surgery. ${ }^{18}$ It resembles the clinical outcome of adult respiratory disease syndrome ${ }^{19}$ and septic shock, ${ }^{18-21}$ results in prolonged stay in intensive care, and is a major cause of death. We investigated whether removal of allogeneic leucocytes reduces postoperative complications in patients undergoing major surgery.

\section{Methods}

Pilot study

We retrospectively analysed all patients with a diagnosis of multiorgan failure who died postoperatively in the intensive care unit at Leiden University Medical Centre between 1988 and 1994. We identified five main surgery groups (aortic aneurysm, major oncological surgery, colorectal surgery, trauma, and pancreatitis). Subsequently a single blinded pilot study stratified for type of surgery was performed in 119 patients. Patients were randomised to receive either standard red cells with the buffy coat removed or filtered leucodepleted red cells. We excluded the surgery types "trauma" and "pancreatitis" because of small numbers. Of the 115 remaining patients, 31 did not receive blood transfusions. Of the 84 transfused patients, $13 / 44(29.5 \%)$ in the non-filtered group and in $9 / 40(22.5 \%)$ in the filtered group experienced multi-organ failure. The overall mortality was $18 \%$ and $10 \%$, respectively. None of the results showed a significant difference.

This pilot showed that a randomised trial was feasible and helped with the study design and calculation of the sample size. On the basis of the pilot study results, we estimated that we would need 400 evaluable transfused patients in each group to detect a 
reduction of $8 \%$ in mortality and of two days' stay in intensive care $(\beta=0.1$ and $\alpha<0.05)$. We assumed that at least $65 \%$ of the patients would be evaluable and receive transfusions and therefore calculated that about 1250 patients had to be randomised.

\section{Study design and participants}

The study was conducted between June 2000 and December 2001 at 19 hospitals in the Netherlands. The primary outcome measures were mortality in hospital and duration of stay in intensive care. The secondary end points were incidence of multi-organ failure and postoperative infections and length of hospital stay.

Transfusions, antibiotic prophylaxis, and use of cell saver were indicated according to hospital protocols. Written informed consent was obtained from all participating patients. Statistical analysis of the study population was performed according to intention to treat ${ }^{162}$ followed by analysis according to transfusion. ${ }^{23}$

\section{Procedures}

Each hospital was invited to assign patients for one or more of the three surgery groups: ruptured aortic aneurysm, elective non-ruptured aortic aneurysm surgery, and gastrointestinal oncology. Aneurysm patients were selected from those undergoing open aorta reconstruction surgery, including transabdominal aortic aneurysm repair and open aorta repair of thoracic aneurysms. Gastrointestinal procedures included resections of stomach, oesophagus, liver, colon, rectum, or pancreas and isolated liver perfusions. We excluded patients who were aged under 18 years, had received transfusions in the three months before the date of randomisation, or had had a previous adverse reaction to blood transfusions or had specific indications for filtered transfusions. In each hospital patients were stratified on the basis of type of surgery. Depending on the hospital, randomisation was performed either by telephone (central registration of randomisation) or by opening numbered and sealed envelopes at the hospital blood transfusion services. The transfusion service ensured that the released red blood cells appeared identical.

\section{Data collection}

We systematically scored characteristics of the patients before, during, and after surgery. The postoperative situation was assessed with standardised queries, daily in intensive care and weekly on the ward until the last day in hospital. Medical records were analysed for postoperative multi-organ failure according to a modified method of Knaus et al. ${ }^{24}$ We defined multi-organ failure as the presence of two or more organs failing or needing support, or both, on the same day. Postoperative infection in patients was scored as defined by the criteria of the Centers for Disease Control and Prevention..$^{25}$ A research team coordinated entry of medical data by using the multicentre data management system ProMISe, on the website of the department of Medical Statistics of the Leiden University Medical Center. All the paper forms with medical data were transferred to the database, either at location via secure internet connections or centrally by the research nurses. Neither the identity of the patient nor the randomisation group was stored in the main database. The actual randomisation was provided to the statistician only at the final analysis.

\section{Transfusion products}

Units of red blood cell concentrates with the buffy coat removed contained up to $8 \times 10^{8}$ white blood cells/unit, whereas in red blood cells filtered before storage the maximum count was $10^{6}$ white blood cells/unit. According to quality control of the blood supply these numbers are accurate in more than $95 \%$ of the units. Registrations of numbers of units that were transfused before, during, and after surgery transfused were downloaded from the hospital blood transfusion service computer system. During the study, protocol violations were reported monthly to the national trial office. Patients who received products in violation of randomisation remained in the assigned arm for intention to treat analysis. In cases of discrepancies between the registration according to the transfusion service and the patients' medical records, we used the transfusion service registration. We performed subgroup analysis of the transfused population, according to transfusion, to evaluate effects related to transfusion (leucocyte dose). For the analysis according to transfusion we excluded 51 patients, who received a total of 404 units, because they received at least one unit of the wrong (not randomised) product. This was mainly caused by non-availability of enough compatible randomised red blood cells but did not disturb the randomised distribution in the numbers of transfusions and patients transfused. Therefore, the number of patients analysed according to transfusion $(\mathrm{n}=494)$ differed from the number of transfused patients analysed according to intention to treat $(\mathrm{n}=545)$. In the analysis according to transfusion, we compared patients who received 1-3, 4-10, or $>10$ units.

\section{Statistical analysis}

An independent data safety committee performed interim analysis of the end points (death, duration of stay in intensive care, and multi-organ failure) in 416 included patients. No objections were raised against continued recruitment.

We performed univariate comparison of baseline qualitative variables by $\chi^{2}$ test; for quantitative parameters we used $t$ test or Mann-Whitney U test. For comparison of outcome measures in both study groups, we stratified patients by participating hospitals and the three major types of surgery. Whenever the outcome was dichotomous, we used the Mantel-Haenszel approach. For continuous variables, we used analysis of variance, normalising the outcome (with a log function) if necessary. In the case of dichotomous outcome measures (mortality, multi-organ failure, and incidence of infection), we estimated the difference between the groups as odds ratios with $95 \%$ confidence intervals. Firstly, common odds ratios were calculated for each of the three types of surgery (averaged over the participating centres). If the result of the standard test for heterogeneity was insignificant, we estimated a final common odds ratio over the three types of surgery as the final effect size. If the odds ratios of the three types were significantly different, we have shown them for each type. Although we have shown their weighted average (the common odds ratio), it should be noted that the estimate depends intrinsically on the relative sizes of the contributing surgical type. We used bootstrapping ${ }^{26}$ to test the equality of variances in the mean differences in the mean length of stay in intensive care and in hospital.

\section{Results}

Over 15 months, 1200 patients were randomised in 19 hospitals (fig 1); 595 patients were randomised to filtered products and 605 to non-filtered products. We excluded 149 patients: 69 patients who received other diagnoses during or after surgery, 44 patients whose surgery was cancelled (or it took place in a nonparticipating hospital), 14 patients who declined to take part, and 22 patients because of administrative and logistic errors. The intake of patients in the study had to be stopped at the end of 2001 because of the implementation of universal leucocyte 


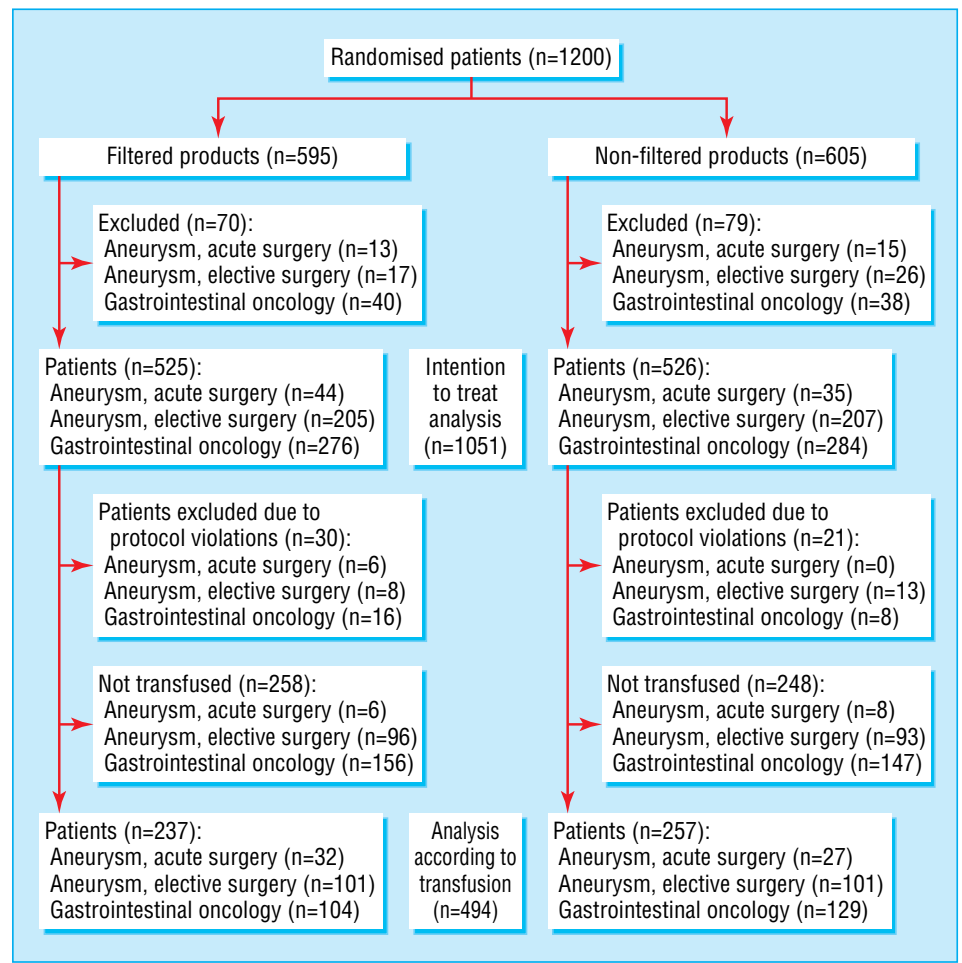

Fig 1 Trial profile

depletion of red blood cells in the Netherlands. This measure was taken by the Dutch Ministry of Health in an effort to reduce the risk of possible transmission of variant Creutzfeldt-Jacob disease in non-filtered transfusions.

\section{Patients' characteristics}

There were 1051 patients eligible for analyses (526 in the non-filtered group and 525 in the filtered arm): 79 acute aneurysm surgery, 412 elective aneurysm surgery, and 560 gastrointestinal oncological surgery. Table 1 shows the characteristics of the patients. The average duration of surgery was 208 minutes, though in 31 patients the exact duration was not known. Ninety eight patients (9\%) died in hospital (28 acute aneurysm; 36 elective aneurysm, and 34 gastrointestinal surgery). Two thirds of the patients $(n=688)$ stayed in intensive care. The intensive care record was not recovered for nine patients. The median stay in intensive care (25th-75th centiles) for the total population was 2 (0-3) days (5 (2-18), 2 (2-4), and 1 $(0-2)$ days for the three types of surgery, respectively). Multi-organ failure occurred in 164 (16\% patients (43 (54\%), 74 (18\%), and $47(8 \%)$, respectively). We had information about postoperative infection in 1011 of the 1051 patients. Postoperative infections occurred in 244 (24\%) patients of the evaluable study population (34 (45\%), 77 (19\%), and 133 (25\%), respectively). The median duration of hospital stay was 11 days (25th-75th centiles: 8-17 days). Two thirds (700 patients) of the total patient population stayed in hospital for less than 15 days, a fifth (210 patients) stayed 15-30 days, and 10 (1\%) stayed for more than 90 days. The median durations of hospital stay (25th75 th centiles) for the three types of surgery were 17 (10-28), 10 (7-15), and 12 (9-17) days, respectively.

\section{Number of transfusions}

In the transfused population analysed according to intention to treat, 278 patients in the group randomised to non-filtered products and 267 patients in the group randomised to filtered products received transfusions. The median number (25th-75th centiles) of transfusions in the total randomised, intention to treat population was lower than expected ( 1 unit (0-4 units), table 1). In the population analysed according to transfusion 3013 units were administered to 494 patients with a median transfused dose of 4 (2-6) units/patient (table 1).

\section{Intention to treat analysis}

Table 2 shows the results of the intention to treat analysis for the primary and secondary end points.

\section{Primary end points}

Mortality in hospital-Mortality in hospital was $10.3 \%$ (54 patients) in the non-filtered group and 8.4\% (44 patients) in the filtered group (common odds ratio $0.80,95 \%$ confidence interval 0.53 to 1.21 ). The results of the test for heterogeneity among the three types of surgery was not significant $(P=0.20)$, indicating that the odds ratios for specific types of surgery do not actually differ from the common odds ratio for the total study population. However, there was a significant reduction in mortality $(0.47,0.23$ to 0.99$)$ in favour of filtered products in the patients undergoing gastrointestinal surgery. Because this difference in mortality between 23 (non-filtered) $v 11$ patients (filtered) $(8 \% v 4 \%)$ could have been caused by imbalanced randomisation with respect to age, sex, duration of surgery, number of blood transfusions, or type of surgery, we have described the gastrointestinal group in more detail in table 3.

Stay in intensive care-There was no significant difference in stay in intensive care between the randomised groups, though in the filtered group patients stayed an average of 0.4 days less ( -1.6 to 0.6 days).

\section{Secondary end points}

Multi-organ failure-One hundred and sixty four patients experienced multi-organ failure (16\% of the total study population). There was a significantly lower incidence in the filtered group. 
Table 1 Characteristics of study population according to allocation to transfusions with filtered or non-filtered red blood cells and analysed group

\begin{tabular}{|c|c|c|c|c|}
\hline Analysis group & $\begin{array}{c}\text { No of } \\
\text { patients }\end{array}$ & $\begin{array}{l}\text { Total in analysis } \\
\quad(\mathrm{n}=1051)\end{array}$ & $\begin{array}{c}\text { Non-filtered } \\
(\mathrm{n}=526)\end{array}$ & $\begin{array}{l}\text { Filtered } \\
(n=525)\end{array}$ \\
\hline \multicolumn{5}{|l|}{ No (\%) of women } \\
\hline Total (intention to treat) & 1051 & $326(31)$ & $163(31)$ & $168(32)$ \\
\hline Acute aortic aneurysm (AA) & 79 & $8(10)$ & $11(14)$ & $5(7)$ \\
\hline Elective aortic aneurysm (EA) & 412 & $82(20)$ & $78(19)$ & $82(20)$ \\
\hline Oncological Gl surgery (GI) & 560 & $241(43)$ & $230(41)$ & $252(45)$ \\
\hline \multicolumn{5}{|l|}{ Mean (SD) age (years) } \\
\hline Total & 1051 & $66(11.2)$ & $67(11.0)$ & $66(11.5)$ \\
\hline$\overline{\mathrm{AA}}$ & 79 & $71(8.5)$ & $70(9.6)$ & $71(7.4)$ \\
\hline$\overline{E A}$ & 412 & $66(10.5)$ & $67(9.9)$ & $66(11.1)$ \\
\hline$\overline{\mathrm{Gl}}$ & 560 & $66(12.0)$ & $66(11.8)$ & $65(12.1)$ \\
\hline \multicolumn{5}{|l|}{ No $(\%)$ of patients transfused } \\
\hline Total & 1051 & $545(52)$ & $278(53)$ & $267(51)$ \\
\hline $\mathrm{AA}$ & 79 & $65(82)$ & $27(77)$ & $38(86)$ \\
\hline$\overline{E A}$ & 412 & $223(54)$ & $114(55)$ & $109(53)$ \\
\hline Gl & 560 & $257(46)$ & $137(48)$ & $120(43)$ \\
\hline \multicolumn{5}{|c|}{ Median units/patient (25th-75th centiles) } \\
\hline Total & 1051 & $1(0-4)$ & $1(0-4)$ & $1(0-4)$ \\
\hline$\overline{\mathrm{AA}}$ & 79 & $7(2-14)$ & $6(2-14)$ & $7(2-14)$ \\
\hline EA & 412 & $2(0-4)$ & $1(0-4)$ & $2(0-4)$ \\
\hline GI & 560 & $0(0-2)$ & $0(0-2)$ & $0(0-2)$ \\
\hline \multicolumn{5}{|c|}{ Median units/patient (25th-75th centiles) in population analysed by transfusion } \\
\hline Total & 494 & $4(2-6)$ & $4(2-6)$ & $4(2-7)$ \\
\hline$\overline{\mathrm{AA}}$ & 59 & $8(3-15)$ & $8(4-15)$ & $8(2-15)$ \\
\hline EA & 202 & $4(2-6)$ & $4(2-6)$ & $4(2-7)$ \\
\hline GI & 233 & $3(2-6)$ & $3(2-4)$ & $2(2-4)$ \\
\hline \multicolumn{5}{|l|}{ Mean duration of surgery $(\mathrm{min})^{\star}$} \\
\hline Total & 1020 & 208 & 210 & 205 \\
\hline$\overline{\mathrm{AA}}$ & 73 & 195 & 201 & 190 \\
\hline EA & 402 & 230 & 228 & 232 \\
\hline$\overline{\mathrm{Gl}}$ & 545 & 193 & 198 & 188 \\
\hline
\end{tabular}

*Duration of surgery was missing for 31 patients.

The overall mean odds ratio for incidence of multi-organ failure was $0.70(0.49$ to $0.99 ; \mathrm{P}=0.05)$.

Postoperative infections - The incidence of postoperative infections was similar in the two groups. The combined odds ratio for the three types of surgery was 0.98 (0.73 to 1.32). Table 2 and figures 2 and 3 show the odds ratios for the various groups of patients.
Hospital stay-In the total patient group, those randomised to filtered products stayed an average of 2.4 days less (4.8 to 0.0 day). Figure 4 shows the length of stay in the total population and in those patients undergoing elective surgery for aneurysm.

\section{Analysis according to transfusion}

Of the 545 transfused patients, we excluded 51 from analysis according to transfusion because of protocol violations. Within the total population analysed according to transfusion $(\mathrm{n}=494)$, the result of the test for heterogeneity among the surgical groups was not significant for mortality, multi-organ failure, and infection. The overall mortality in hospital in the patients analysed according to transfusion was $15 \%$. Neither in the patients analysed according to transfusion $(0.74,0.44$ to 1.24$)$ nor in the three types of surgery was there a significant difference for in hospital mortality between the randomisation groups. In the gastrointestinal patients the confidence interval became wider than the corresponding intention to treat estimate and covered the value of 1.0, although the point estimate itself was similar $(0.53,0.17$ to 1.25$)$.

The median stay in intensive care (25th-75th centile) of the patients analysed according to transfusion was 2 days (1- 6 days). There were no differences in mean length of stay between the two groups, neither in the total population nor according to type of surgery. The incidence of multi-organ failure in the patients analysed according to transfusion was $26.1 \%$ (129/494). The incidence was lower in the group who received filtered transfusions $(0.76,0.49$ to 1.16$)$, but this difference failed to reach significance $(\mathrm{P}=0.20)$. The mean hospital stay was 4.5 days shorter (23.6 $v 19.1$ days) in the filtered group ( 0.4 to 8.5 days, $\mathrm{P}=0.032$ ), shown as cumulative hospital discharge in figure 4 . For all end points there was no significant association with the number of transfusions given.

\section{Discussion}

In patients undergoing major vascular or oncological surgery, the use of filtered red blood cells for transfusions results in shorter stays in hospital and a lower incidence of multi-organ failure. Major surgery is associated with postoperative complications. We carried out this study because we had previously observed a reduced incidence of postoperative infections and

Table 2 Intention to treat analyses of primary and secondary end points. Figures are totals in group (non-filtered; filtered) and odds ratios or differences in means ( $95 \%$ confidence intervals)

\begin{tabular}{|c|c|c|c|c|}
\hline & Total ITT population & Acute aneurysm & Elective aneurysm & Gastrointestinal oncology \\
\hline No of patients & $1051(526 ; 525)$ & $79(35 ; 44)$ & $412(207 ; 205)$ & $560(284 ; 276)$ \\
\hline \multicolumn{5}{|l|}{ Mortality } \\
\hline No of patients & $98(54 ; 44)$ & $28(11 ; 17)$ & $36(20 ; 16)$ & $34(23 ; 11)$ \\
\hline Odds ratio & 0.80 (0.53 to 1.21$)$ & 1.37 (0.54 to 3.51$)$ & 0.79 (0.40 to 1.57$)$ & $0.47^{\star}$ (0.23 to 0.99$)$ \\
\hline \multicolumn{5}{|c|}{ Intensive care unit stayt } \\
\hline Mean (days) & $4.3(4.5 ; 4.1)$ & $12.2(10.5 ; 13.6)$ & $4.7(5.3 ; 4.1)$ & $2.9(3.1 ; 2.7)$ \\
\hline Difference & $-0.4(-1.63$ to 0.61$)$ & 3.0 (-3.5 to 9.6$)$ & $-1.2(-2.88$ to 0.40$)$ & $-0.5(-1.33$ to 1.00$)$ \\
\hline \multicolumn{5}{|l|}{ Hospital stay } \\
\hline Mean (days) & $16.8(17.9 ; 15.6)$ & $22.2(22.9 ; 21.6)$ & $14.3(15.7 ; 13.0)$ & $17.8(19.0 ; 16.5)$ \\
\hline Difference & $-2.4^{*}(-4.75$ to 0.005$)$ & $-1.3(-10.5$ to 7.9$)$ & $-2.7(-5.7$ to 0.3$)$ & $-2.4(-6.1$ to 1.2$)$ \\
\hline \multicolumn{5}{|c|}{ Multi-organ failureł } \\
\hline No of patients & $164(91 ; 73)$ & $43(19 ; 24)$ & $74(42 ; 32)$ & $47(30 ; 17)$ \\
\hline Odds ratio & $0.70^{\star}(0.49$ to 0.999$)$ & 1.01 (0.41 to 2.46$)$ & 0.73 (0.44 to 1.21$)$ & 0.56 (0.30 to 1.03$)$ \\
\hline \multicolumn{5}{|l|}{ Infection§ } \\
\hline No of patients & $244(121 ; 123)$ & $34(15 ; 19)$ & $77(41 ; 36)$ & $133(65 ; 68)$ \\
\hline Odds ratio & 0.98 (0.73 to 1.32$)$ & 0.95 (0.38 to 2.37$)$ & 0.86 (0.52 to 1.42$)$ & 1.07 (0.73 to 1.59$)$ \\
\hline
\end{tabular}

${ }^{*} \mathrm{P} \leq 0.05$.

$\mathrm{tn}=1042$; data missing for nine patients.

$\ddagger \mathrm{n}=1034$; data missing for 17 patients.

$\S n=1011$; data missing for 40 patients. 
Table 3 Patients undergoing gastrointestinal surgery differentiated by sex, age, type of surgery, blood transfusion with filtered or non-filtered red blood cells, and duration of surgery according to survival

\begin{tabular}{|c|c|c|c|c|c|}
\hline & \multirow[b]{2}{*}{$\begin{array}{l}\text { Total* }^{*} \\
(\mathrm{n}=560)\end{array}$} & \multicolumn{2}{|c|}{ Survived } & \multicolumn{2}{|c|}{ Died $\dagger$} \\
\hline & & $\begin{array}{l}\text { Non-filtered } \\
(n=284)\end{array}$ & $\begin{array}{l}\text { Filtered } \\
(n=276)\end{array}$ & $\begin{array}{l}\text { Non-filtered } \\
(n=23)\end{array}$ & $\begin{array}{r}\begin{array}{c}\text { Filtered } \\
(n=11)\end{array}\end{array}$ \\
\hline \multicolumn{6}{|l|}{ Sex: } \\
\hline Male & 318 & 167 & 151 & 12 & 9 \\
\hline Female & 242 & 117 & 125 & 11 & 2 \\
\hline \multicolumn{6}{|l|}{ Age (years): } \\
\hline$<60$ & 174 & 88 & 86 & 5 & 2 \\
\hline $60-70$ & 179 & 90 & 89 & 5 & 5 \\
\hline$>70$ & 207 & 106 & 101 & 13 & 4 \\
\hline \multicolumn{6}{|c|}{ Units of red blood cells transfused: } \\
\hline 0 & 303 & 147 & 156 & 5 & 3 \\
\hline $1-3$ & 137 & 75 & 62 & 6 & 1 \\
\hline $4-10$ & 90 & 44 & 46 & 7 & 3 \\
\hline$>10$ & 30 & 18 & 12 & 5 & 4 \\
\hline \multicolumn{6}{|c|}{ Duration of surgery (mins): } \\
\hline$<120$ & 172 & 81 & 91 & 6 & 3 \\
\hline $120-179$ & 162 & 87 & 75 & 9 & 2 \\
\hline $180-239$ & 115 & 59 & 56 & 3 & 2 \\
\hline $240-359$ & 76 & 37 & 39 & 4 & 4 \\
\hline$\geq 360$ & 35 & 20 & 15 & 1 & 0 \\
\hline \multicolumn{6}{|l|}{ Type of surgery: } \\
\hline Upper Gl surgery $\ddagger$ & 131 & 60 & 71 & 6 & 5 \\
\hline Laparotomy & 26 & 16 & 10 & 2 & 1 \\
\hline Colectomy & 118 & 64 & 54 & 5 & 1 \\
\hline $\begin{array}{l}\text { Rectal (sigmoid) } \\
\text { resections }\end{array}$ & 285 & 144 & 141 & 10 & 4 \\
\hline
\end{tabular}

*Equal distributions.

†Unequal distributions between non-filtered and filtered products.

łIncludes resections of oesophagus, gastrectomy, liver surgery, jejunectomy,

pancreatico-duodenectomy.

death due to multi-organ failure in patients undergoing cardiac surgery who had received 4 or more units of filtered rather than non-filtered red blood cells. ${ }^{17}$ Because our previous study could not discriminate between the interaction of the large number of transfusions and the long period of extra-corporal circulation, in the present study we selected other categories of patients with a high probability for both multiple transfusions and multi-organ failure. We selected acute ruptured and elective aortic aneurysm and major gastrointestinal surgery on the basis of retrospective analysis and on a pilot study of patients at risk of multi-organ failure and death after surgical intervention. We analysed data according to type of surgery because of differences in surgical

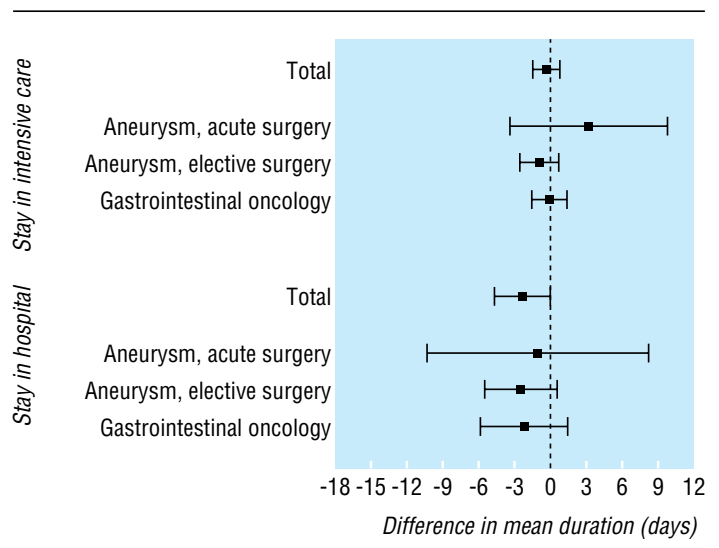

Fig 2 Intention to treat analysis of primary and secondary end points. Effects on stay in intensive care and in hospital reported as difference in means $(95 \% \mathrm{Cl}$ of difference)

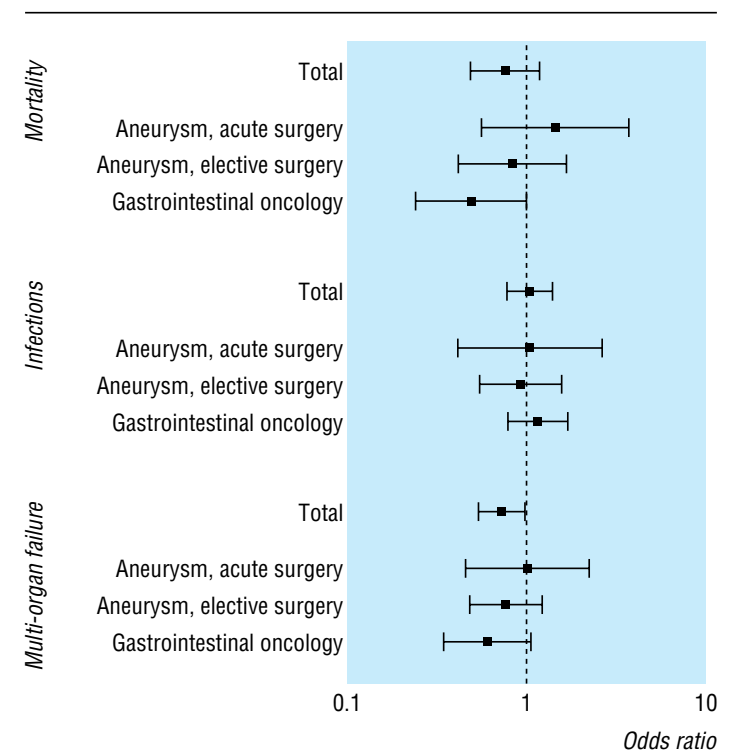

Fig 3 Intention to treat analysis of primary and secondary end points. Effects on mortality, multi-organ failure, and infection depicted as odds ratio (filtered/non-filtered) with $95 \%$ confidence intervals

procedures, ischaemia, and patients' characteristics and thus in potential risks for postoperative complications.

We hoped to provide decision makers with evidence regarding a universal leucocyte filtration programme. The study ended early because politicians decided to mandate universal leucocyte filtration of red blood cells in the Netherlands to reduce the risk of transmission of prions through non-filtered transfusions. However, this meant that we had to stop recruitment before we reached our planned study size.

\section{End points}

Analysis of the total study population according to randomisation (intention to treat) showed no significant differences in the primary end points (mortality and stay in intensive care). With respect to secondary end points, we found a significant difference in the incidence of postoperative multi-organ failure in favour of filtered products. The incidences of postoperative infections were not significantly different between the two randomised groups. The mean hospital stay was 2.4 days shorter in the group randomised to filtered products. This effect on hospital stay was more profound in those patients randomised who actually received transfusion, with a mean reduction of 4.5 days. When we looked at patients who received $0,1-3,4-10$, or $>10$ units of red blood cells we found no dose-dependent difference between the randomisation groups. The actual number of transfused patients in this trial was 545 , and only 254 patients received 4 or more units. Both the size of the total transfused population and the number of patients transfused with more than 3 units, may have been too small to detect a dose effect. ${ }^{27}$

Although we found no significant benefit of filtered products on mortality in hospital in the total population, we did see an effect in the subgroup of patients undergoing gastrointestinal surgery. We excluded an apparent imbalance in randomisation (which could have caused this difference) by showing that with respect to relevant risk factors the distribution of randomised gastrointestinal patient to non-filtered and filtered products was balanced (table 3). This observation is in agreement with the outcomes of a recent randomised controlled trial in cardiac surgery ${ }^{28}$ and of the Canadian registration study. ${ }^{29}$ These studies showed that compared with transfusions of non-filtered red 

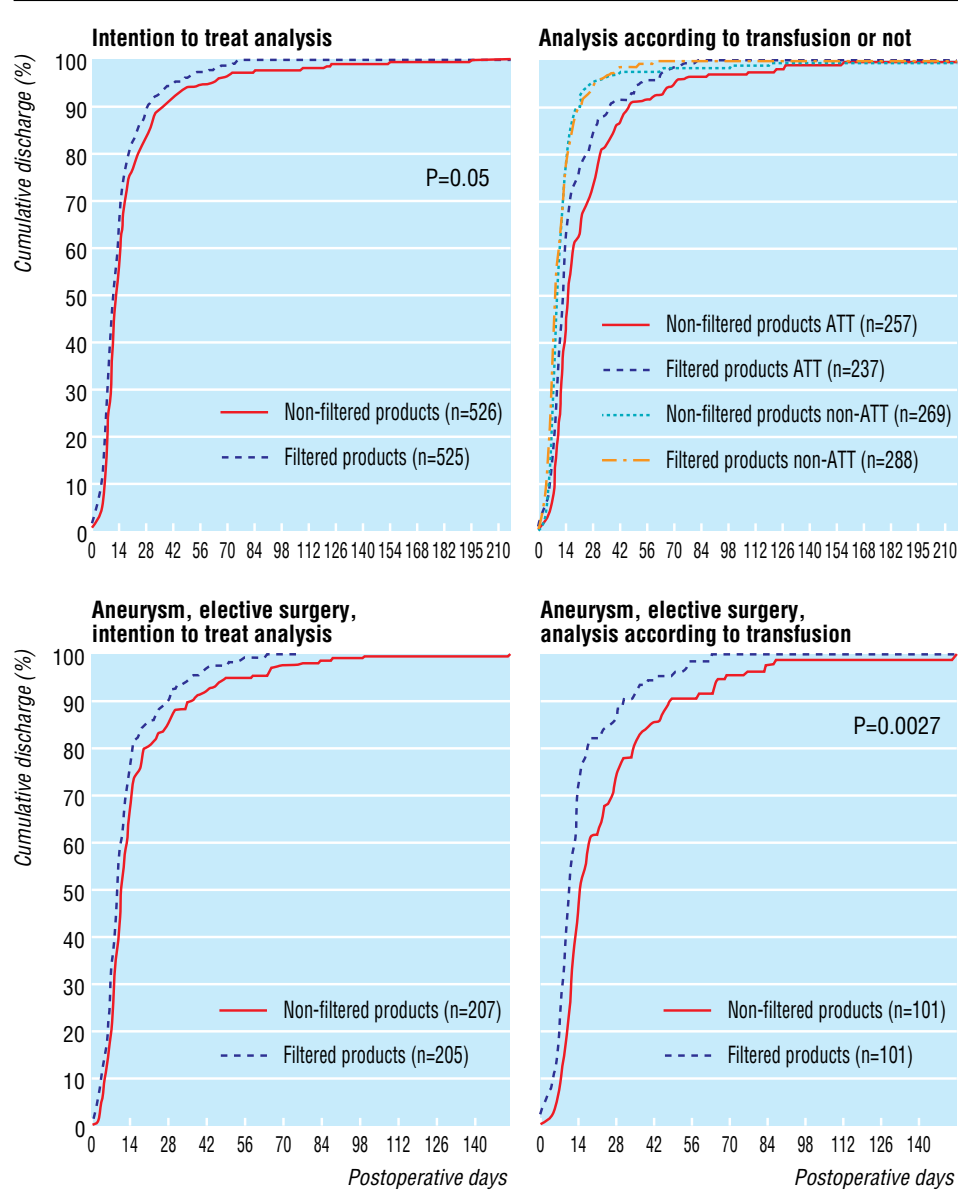

Fig 4 Cumulative hospital discharges, calculated by analysis of length of hospital stay in all patients and in those who received transfusion, according to various subgroups

blood cells, transfusions of filtered products can lead to a small reduction in mortality in hospital in patients undergoing cardiac and orthopaedic surgery and in trauma patients.

We found no evidence to support the use of filtered products to prevent infection in patients undergoing major surgery. In our three previous studies that compared filtered products with red blood cells with the buffy coat removed (the standard Dutch product), we found a reduction in postoperative infections only in patients undergoing cardiac surgery who had received more than 3 units. ${ }^{11}{ }^{18}$ In our previous (multicentre) non-cardiac surgery study $^{11}$ and in the present study only a relatively small number of patients received 4 or more units.

Another factor that may account for the discrepancies between the randomised controlled trials is the multicentre or single centre design of the studies. The reduced incidence of postoperative infections associated with leucocyte filtration is most often shown, albeit with a large variation in magnitude, in randomised controlled single centre studies, ${ }^{13-15}{ }^{17}$ and it is seldom found in multicentre trials. ${ }^{16}$ Differences in hospital policies, individual surgeons, heterogeneity of patients, and efforts in registration (the Centers for Disease Control and Prevention gives only positive criteria for definition of infections) all contribute strongly to differences in the (reported) incidence of postoperative infections. In multicentre trials, the centres with a fewer patients are more affected by these variables. From our 19 participating hospitals, four hospitals had included more than 90 patients each. Within these four hospitals the single centre odds ratio ranged from $0.91(0.32$ to $2.53 ; \mathrm{n}=124)$ to 1.76 ( 0.85 to
3.66; $\mathrm{n}=153$ ). Within the 15 hospitals that had included fewer than 90 patients, the single centre odds ratios ranged from 0.07 ( 0.01 to $0.58 ; \mathrm{n}=39)$ to $6.75(0.93$ to $49.26 ; \mathrm{n}=21)$.

These results illustrate that an identical study design may come up with opposing effects in different small hospitals (some effects even showing significance) and no effect in the larger hospitals or combined analyses. A large retrospective, nonrandomised, multicentre intervention study in Canada also found no effect of filtered products on postoperative infections. ${ }^{29}$ This study comprised about 15000 patients in intensive care who had each received a mean of 3.5 units of red blood cells. In our study, our analysis of the common odds for all the end points, including infection, in the 19 centres showed significant variation between hospitals for infection. This is explained by two centres, with a significant difference between the trial groups, in which the average inclusion rates were lower than six patients a month. However, we did not see this significant variation in infection in the four hospitals that included more than 90 patients, with average inclusion rates of more than six patients a month. We found no other significant heterogeneity between the centres with respect to the other end points.

The effect of leucocyte filtration of red blood cells on incidence of multi-organ failure did reach significance in the intention to treat population, but significance was lost in the smaller analysis according to transfusion. Another benefit of filtered products in our study was a reduction in mean length of hospital stay. The reduction in hospital stay was partly due to fewer patients who received filtered products who stayed for 


\section{What is already known on this topic}

Reduction of leucocytes in red blood cell concentrates by filtration results in less alloimmunisation in patients receiving transfusions and transplants

In patients undergoing cardiac surgery and transfused with at least 4 units the use of filtered red blood cells reduces postoperative infections and mortality

The use of filtered red blood cells has also been shown to reduce postoperative infections in patients undergoing colorectal surgery

\section{What this study adds}

The use of filtered red blood cells results in a shorter stay in hospital and a lower incidence of multi-organ failure in patients undergoing major vascular or oncological surgery

Mortality was lower in the subgroup of patients undergoing gastrointestinal oncological surgery

more than 90 days in hospital (10 non-filtered $v 1$ filtered). The reduction in hospital stay was present in all subgroups and most pronounced in the patients undergoing elective surgery for aneurysm who received transfusions. The two Canadian intervention studies also showed a reduction in hospital stay. ${ }^{29} 30$

\section{Economic evaluation}

Reduction in hospital stay supports the general leucocyte reduction of red blood cells by filtration. If we extrapolate the benefit of filtrated products to the Dutch healthcare system, with 16300 aneurysm and gastrointestinal procedures a year, a mean reduction of 2.4 days in hospital would reduce the national hospital costs by $€ 29.5 \mathrm{~m} /$ year $(£ 19.6 \mathrm{~m} ; \$ 35.0 \mathrm{~m})$. In the Netherlands the annual cost of universal leucocyte depletion of red blood cells is about $€ 20 \mathrm{~m}$ (assuming filtration costs of $€ 40 /$ unit). The reduced mean hospital stay associated with filtered products could, to a large extent, compensate the extra cost of filtration in the Netherlands. In other countries where standard whole blood transfusion has been compared with filtered red blood cells, implementation of filtration seems to be cost neutral or cost saving in some settings. ${ }^{27} 31$

\section{Conclusion}

In conclusion, although we had to stop recruiting patients before we reached the desired study size, results for some end points had reached significance. Leucocyte reduced transfusions in this group of patients undergoing major surgery significantly reduced the incidence of multi-organ failure and length of hospital stay. Our results also contribute to the discussion of the cost effectiveness of using filtered red blood cells.

We thank the following members of the Tactics trial group for their participation: M S Harvey, R A E M Tollenaar (Leiden University Medical Centre); P J Geneste, G A E Ponjee (Reinier de Graaf Groep, Delft); C M A Bruijninckx, H Schonewille, M R Schipperus (Leyenburg ziekenhuis, Hague); J C A de Mol van Otterloo, J A Swen, W Huisman (Medisch Centrum Haaglanden, Hague); J F W B Rijksen, R W L M (Niessen Rijnland ziekenhuis, Leiderdorp); A B Bijnen (Medisch Centrum Alkmaar); H Rijna, C H Noordzij (Kennemer Gasthuis, locaties Elisabeth Gasthuis and Deo, Haarlem and locatie Zeeweg, IJmuiden); T I F M Bloemen, F L A Willekens (Ziekenhuis Rijnstate, Arnhem); W B Barendregt, K van Beugen (Canisius-Wilhelmina Ziekenhuis, Nijmegen); M T C Hoedt, S T IJpma (Albert Schweitzer, locatie Dordwijk, Dordrecht); R M Weghorst (Medisch Spectrum Twente Enschede); P J G Jörning, J J C M van der Leur (Isala Klinieken, locatie Sophia, Zwolle); R J T J Welten, M J M de Groot (Atrium Medisch Centre
Heerlen); N A Foudraine, J M W H van Gend (St. Maarten's Gasthuis, Venlo); E C M van Pampus (Academic Hospital Maastricht); F J L M Haas, B van Ramshorst, F L Moll (St. Antonius ziekenhuis, Nieuwegein); J D Blankensteijn, A Schuurhuis, H C van Prooijen (University Medical Center Utrecht); G P Gerritsen, C J H F Doensen-Ydema (Tweesteden Ziekenhuis, Tilburg); G W T Janssen (St. Joseph Ziekenhuis, Veldhoven).We also thank all contributors from the Sanquin Blood Bank, the research nurses, and members of the data monitoring safety committee of Tactics.

Contributors: AB, JK, JHvB, CJHvdV, and LMGvdW developed the study concept and design. LMGvdW performed the pilot study. AB and JAvH wrote the grant application, obtained funding for the study, and drafted the manuscript. JAvH recruted the 19 local coordinators for enrolment of the patients and acquisition of the data. RHG, AWMMKvG, RMJW, RMHR, and JK recruited and enrolled more than 75 evaluable patients for data acquisition in their hospitals and provided critical remarks for important intellectual content of the manuscript. $\mathrm{AB}, \mathrm{JK}, \mathrm{JHvB}$, and $\mathrm{CJHvdV}$ were principal study supervisors. JAvH and LMGvdW analysed the data and contributed important intellectual content in interpretation of the data by the study supervisors. RB provided the data management infrastructure (via secure internet) as well as the statistical analyses and $\mathrm{WBvdH}$ provided critical remarks and performed the cost analysis of filtered products. All authors approved the final version of the manuscript. JAvH is guarantor.

Funding: Health Insurance Board (College voor Zorgverzekeringen (project OG99/023)), the Netherlands. The National Sanquin Blood Banks produced blood products under study and reimbursed the difference in costs.

Competing interests: None declared.

Ethical approval: The protocol was first approved by the ethics committee of the Medical Spectrum Twente recognised by Dutch Central Council for Medical Research (CCMO) and subsequently endorsed by all ethics committees of the other participating hospitals.

1 Wobbes T, Bemelmans BL, Kuypers JH, Beerthuizen GI, Theeuwes Ag. Risk of postoperative septic complications after abdominal surgical treatment in relation to perioperative blood transfusion. Surg Gynecol Obstet 1990;171:59-62.

2 Pinto V, Baldonedo R, Nicolas C, BPreez A, Aza J. Relationship of transfusion and infectious complications after gastric carcinoma operation. Transfusion 1991;31:114-8. Spark JI, Chetter IC, Kester RC, Scott DJ. Allogeneic versus autologous blood during abdominal aortic aneurysm surgery. EurJ Vasc Endovasc Surg 1997;14:482-6.

4 Hébert PC, Yetisir E, Martin C, Blajchman MA, Wells G, Marshall J, et al. Is a low transfusion threshold safe in critically ill patients with cardiovascular diseases? Crit Care Med 2001;29:227-33

5 Duffy G, Neal KR. Differences in postoperative infection rates between patients receiving autologous and allogeneic blood transfusion: a meta-analysis of published ing autologous and allogeneic blood transfusion: a meta-analysis
randomised and nonrandomised studies. Transfusion Med 1996;6:325-8.

6 Vamvakas EC, Blajchman MA. Deleterious clinical effects of transfusion-associated immunomodulation: fact or fiction? Blood 2000;97:5:1180-95.

7 Sharma AD, Sreeram G, Erb T, Grocott HP, Slaughter TF. Leukocyte-reduced blood transfusions: Perioperative indications, adverse effects and cost analysis. Anesth Analg 2000;90:1315-23.

8 Groeneveld AB, Raijmakers PG, Rauwerda JA, Hack CE. The inflammatory response to vascular surgery-associated ischaemia and reperfusion in man: effect on postoperative pulmonary function. Eur J Vasc Endovasc Surg 1997;14:351-9.

9 Rapaport FT, Dausset J. The possible role of leukocyte components in the production of the beneficial effects of blood transfusion in human transplantation. Transplant Proc 1983;15:952-5.

10 Collier AC, Kalish LA, Busch MP, Gernsheimer T, Assmann T, Lane TA Leukocyte-reduced red blood cell transfusions in patients with anemia and human immunodeficiency virus infection. The viral activation transfusion study: a randomised controlled trial. JAMA 2001;285:1592-601.

11 Houbiers JG, Brand A, van de Watering LM, Hermans J, Verwey PJ, Bijnen AB, et al. Randomised controlled trial comparing transfusion of leukocyte-depleted or buffy-coat-depleted blood in surgery for colorectal cancer. Lancet 1994;344:573-8.

12 Titlestad IL, Ebbesen LS, Ainsworth AP, Lillevang ST, Qvist N, Georgsen J. Leukocytedepletion of blood components does not significantly reduce the risk of infectious complications. Results of a double-blinded, randomised study. Int J Colorectal Dis 2001;16:147-53.

13 Jensen LS, Anderson AJ, Christiansen PM, Hokland P, Juhl CO, Madsen G, et al. Postoperative infection and natural killer cell function following blood transfusion in operative infection and natural killer cell function following blood tran
patients undergoing elective colorectal surgery. Br J Surg 1992;79:513-6.

14 Tartter PI, Mohandas K, Azar P. Randomised trial comparing packed red blood cell transfusion with and without leukocyte depletion in gastrointestinal surgery. Am J Surg $1998 ; 176: 462-6$

15 Jensen LS, Kissmeyer-Nielsen P, Wolff B. Randomised comparison of leukocytedepleted versus buffy-coat-poor blood transfusion and complications after colorectal surgery. Lancet 1996;348:841-5.

16 Vamvakas EC. Multicentre randomised controlled trials in transfusion medicine. Transfusion Med Rev 2000;14:137-50.

17 Van de Watering LM, Hermans J, Houbiers JG, van den Broek PJ, Bouter H, Boer F, et al. A beneficial effect of leukocyte depletion of transfused blood on postoperative mortality in patients undergoing cardiac surgery: a randomised clinical trial. Circulation tality in patients
$1998 ; 97: 562-8$.

18 Goris RJ, te Boekhorst TP, Nuytinck JKS, Gimbrere JS. Multiple-organ failure. Generalized autodestructive inflammation? Arch Surg 1985;120:1109.

19 Ware LB, Matthay MA. The acute respiratory distress syndrome. $N$ Engl J Med 2000;342:1334-49. 
20 Nathens AB, Marshall JC. Sepsis, SIRS and MODS: what's in a name? World J Surg 1996;20:386-91

21 Asimakopoulos G. Mechanisms of the systemic inflammatory response. Perfusion 999;14:269-77

22 Moher D, Schulz KF, Altman DG. The CONSORT statement: revised recommendations for improving the quality of report of parallel-group randomised trials. Lancet 2001;357:1191-4.

23 Assmann SF, Pocock SJ, Enos LE, Kasten LE. Subgroup analysis and other (mis)uses of baseline data in clinical trial. Lancet 2000;255:1064-9.

24 Knaus WA, Draper EA, Wagner DP, Zimmerman JE. Prognosis in acute organ system failure. Ann Surg 1985;202:685.

25 Garner JS, Jarvis WR, Emori TG, Horan TC, Hughes JM. CDC definitions for nosocomial infections. Am I Infect Control 1985;16:128-40.

26 Desgagne A, Castilloux AM, Angers JF, LeLorier J. The use of the bootstrap statistical method for the pharmacoeconomic cost analysis of skewed data. Pharmacoeconomic $998 ; 13: 487-97$

27 Blumberg N, Heal JM, Cowles JW, Hicks GL Jr, Risher WH, Samuel PK, et al. Leukocyte-reduced transfusions in cardiac surgery. Results of an implementation trial. Am J Clin Pathol 2002;118:376-81

28 Bilgin YM, van de Watering LMG, Lorinser JE, Versteegh MIM, Eijsman L, van Oers $\mathrm{MHJ}$, et al. The effect of prestorage-leukocyte-depletion of erythrocyte concentrates in cardiac surgery: a double-blind randomised clinical trial. Blood 2001:98(suppl):828-9

9 Hebert PC, Fergusson D, Blajchman MA, Wells GA, Kmetic A, Coyle D, et al Clinical out outcomes following institutions of the Canadian universal leukoreduction program for
red blood cell transfusions. JAMA 2003:289:1941-9.

Fed

Fergusson D, Hebert PC, Lee SK, Walker CR, Barrington KJ, Joseph L, et al. Clinical outcomes following institution of universal leukocyte depletion of blood transfusions for premature infants. JAMA 2003;289:1950-6.

31 Dzik WH, Anderson JK, O'Neill EM, Assmann SF, Kalish LA, Stowell CP. A prospective, randomized clinical trial of universal WBC reduction. Transfusion 2002;42:1114-22. (Accepted 18 March 2004)

doi $10.1136 /$ bmj.38103.735266.55
Department of Immunohaematology and Blood Transfusion, Leiden University Medical Center and Sanquin Blood Supply, PO Box 9600, 2300 RC Leiden, Netherlands

Joost A van Hilten senior research scientist

Anneke Brand professor in transfusion medicine

Leo M G van de Watering senior research scientist

Department of Surgery, Leiden University Medical Center, Leiden

$\mathrm{J}$ Hajo van Bockel professor in surgery

Cornelis $\mathrm{J} \mathrm{H}$ van de Velde professor in surgery

Department of Medical Decision Making, Leiden University Medical Center,

Leiden

Job Kievit professor in medical decision making

Wilbert B van den Hout health econometrist

Department of Medical Statistics, Leiden University, Leiden Ronald Brand statistician

Medisch Spectrum Twente, PO Box 50000, 7500 KA Enschede Robert H Geelkerken surgeon

St Joseph Ziekenhuis, P O Box 7777, 5500 MB Veldhoven Rudi M H Roumen surgeon

St Antonius Ziekenhuis, PO Box 2500, 3430 EM Nieuwegein Ronald MJ Wesselink anaesthetist

Albert Schweitzer, Locatie Dordwijk, PO Box 306, 3300 AH Dordrecht Ankie W M M Koopman-van Gemert anaesthetist

Reinier de Graaf Groep, PO Box 5011, 2600 GA Delft

Jan Koning surgeon

Correspondence to:J van Hilten J.A.van_Hilten@lumc.nl 